\title{
IRF3 signaling pathway serves an important role in poly(I:C)-induced procollagen reduction in human skin fibroblasts
}

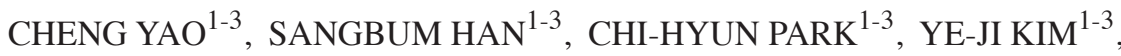 \\ DONG HUN LEE ${ }^{1-3^{*}}$ and JIN HO CHUNG ${ }^{1-4^{*}}$
}

\author{
${ }^{1}$ Department of Dermatology, Seoul National University College of Medicine, Seoul 110-744; ${ }^{2}$ Institute of \\ Human-Environment Interface Biology, Medical Research Center, Seoul National University; ${ }^{3}$ Laboratory of \\ Cutaneous Aging Research, Biomedical Research Institute, Seoul National University Hospital; \\ ${ }^{4}$ Institute on Aging, Seoul National University, Seoul 03080, Republic of Korea
}

Received March 13, 2016; Accepted July 27, 2017

DOI: $10.3892 / \mathrm{mmr} .2017 .8136$

\begin{abstract}
Pattern recognition receptors (PRRs) are part of the immune system. They can recognize pathogen-associated molecular patterns (PAMPs). Toll-like receptors (TLRs) and retinoic acid-inducible gene 1 (RIG-1)-like receptors (RLRs) are 2 types of PRR in the innate immune system. Double-stranded RNA (dsRNA) can exist as a PAMP, including dsRNA viruses. dsRNA is known as a ligand not only for TLR3 but also for RLRs, including melanoma differentiation-associated gene 5 and RIG-1. Collagen is the main structural protein in the extracellular space in the skin. Recently, it was reported that treatment of a synthetic dsRNA, poly(I:C), decreases procollagen expression in skin fibroblasts. However, signaling pathways involved in this process have not yet been fully elucidated. The present study further explored the underlying signaling pathways involved in the processes. It was demonstrated by western blotting that treatment of poly(I:C), but not another PAMP, Pam3CSK4, inhibited procollagen expression in cultured human skin fibroblasts. Treatment of poly(I:C) and Pam3CSK4 induced activation of the mitogen-activated protein kinases and the nuclear factor- $\mathrm{\kappa B}$ pathways. However, only poly(I:C), but not Pam3CSK4, induced the activation of the interferon regulatory factor 3 (IRF3) pathway. By using specific inhibitors, it was demonstrated that inhibition of IRF3 pathway relieved poly(I:C)-induced procollagen reduction. In conclusion, IRF3 signaling pathway serves an important role
\end{abstract}

Correspondence to: Dr Jin Ho Chung or Dr Dong Hun Lee, Department of Dermatology, Seoul National University College of Medicine, 28 Yongon-dong, Chongno-gu, Seoul 110-744, Republic of Korea

E-mail: jhchung@snu.ac.kr

E-mail: ivymed27@snu.ac.kr

*Contributed equally

Key words: poly(I:C), interferon regulatory factor 3, procollagen, fibroblasts in poly(I:C)-induced procollagen reduction in skin fibroblasts. This suggests that the IRF3 signaling pathway may be a key target for collagen regulation in the skin.

\section{Introduction}

Pathogen-associated molecular patterns (PAMPs) are molecules associated with groups of pathogens. PAMPs include molecules from Gram-positive and -negative bacteria, DNA and RNA viruses (1). PAMPs can be recognized by Toll-like receptors (TLRs) and RIG-I-like receptors (RLRs); two types of pattern recognition receptors (PRRs) in the innate immune system $(2,3)$. PAMPs recognized by TLRs and RLRs include lipids, lipoproteins, proteins and nucleic acids derived from bacteria, viruses, parasites and fungi (4). For example, triacyl lipopeptide and diacyl lipopeptide are PAMPs that can be recognized by TLR1/2 and TLR2/6, respectively (5-7). Double-stranded RNA (dsRNA) is a ligand not only for TLR3, but also for RLRs including melanoma differentiation-associated gene 5 (MDA5) and retinoic acid-inducible gene 1 (RIG-I) (8). MDA5 and RIG-I are cytosolic RNA helicases capable of unwinding dsRNA molecules (9). Once recognized by receptors, PAMPs can activate nuclear factor $(\mathrm{NF}) \kappa \mathrm{B}$, activator protein 1 (AP-1), interferon regulatory factor 3 (IRF3), and IRF7 signaling pathways which induce the expression of inflammatory cytokines $(3,10,11)$.

Collagen is the main structural protein in the extracellular space of tissues. Collagen-related diseases can arise from genetic defects or environmental stresses that affect the biosynthesis, assembly, secretion or other processes involved in normal collagen production. Scleroderma results from an overproduction and accumulation of collagen in tissues (12). Skin aging may result from decreased synthesis of collagen and/or induced collagen degradation (13).

Poly(I:C) is a synthetic dsRNA that has frequently been used as a representative dsRNA ligand in numerous studies $(14,15)$. Upon binding to receptors, poly(I:C) is able to selectively activate NF- $\mathrm{B}, \mathrm{AP}-1$ and IRF3 signaling pathways depending on different experiment conditions $(8,16,17)$. It has been suggested that poly(I:C) treatment inhibited procollagen expression by autocrine interferon signaling in skin 
fibroblasts (18). However, the signaling pathways involved in poly(I:C)-induced procollagen reduction have yet to be fully elucidated.

The present study identified that treatment of poly(I:C), but not another PAMP, Pam3CSK4, inhibited procollagen expression in cultured human skin fibroblasts. Although treatment of poly(I:C) and Pam3CSK4 induced activations of the mitogen-activated protein kinases (MAPK) and the NF- $\mathrm{KB}$ pathways, only poly(I:C), not Pam3CSK4, induced the activation of IRF3 pathway. By using two different specific inhibitors, it was identified that inhibition of IRF3 signaling pathway relieved poly(I:C)-induced procollagen reduction in skin fibroblasts.

\section{Materials and methods}

Reagents. Pam3CSK4 and poly(I:C) were purchased from InvivoGen (San Diego, CA, USA) and Tank binding kinase 1 (TBK1) inhibitor BX795 from Calbiochem (EMD Millipore, Billerica, MA, USA). Another TBK1 inhibitor, SU6668, was purchased from Tocris Bioscience (Bristol, UK). For detecting procollagen protein, monoclonal anti-type I procollagen aminoterminal extension peptide antibody (clone SP1.D8; Developmental Studies Hybridoma Bank, Iowa City, IA, USA) was diluted 1:10 in TBST to be used. Antibody for matrix metalloproteinase-1 (MMP-1) was made by Lab Frontier Co., Ltd. (Seoul, Korea). Antibody for $\beta$-actin (cat. no. sc-1616) was purchased from Santa Cruz Biotechnology, Inc. (Dallas, TX, USA). Antibodies for phosphorylated-extracellular signal-regulated kinase (p-ERK)1/2 (cat. no. 9101), p-c-Jun N-terminal kinases (p-JNK) (cat. no. 9251), p-p38 (cat. no. 9211), p-nuclear factor of $\kappa$ light polypeptide gene enhancer in B-cells inhibitor, $\alpha$ (p-IкB- $\alpha$ ) (cat. no. 9246), and p-IRF3 (cat. no. 4947) were purchased from Cell Signaling Technology Inc. (Danvers, MA, USA). Horseradish peroxidase-conjugated anti-mouse (cat. no. sc-2031), anti-rabbit (cat. no. sc-2030) or anti-goat (cat. no. sc-2020) IgG (Santa Cruz Biotechnology, Inc.) were used as secondary antibodies. Primary antibodies other than type I procollagen antibody were diluted 1:1,000 and secondary antibodies were diluted 1:10,000 in TBST for western blotting.

Cell culture. From December 2013 to January 2014, three young healthy volunteers provided foreskin samples at the Department of Dermatology, Seoul National University Hospital (Seoul, Korea). Human foreskin fibroblasts from young healthy volunteers were cultured in Dulbecco's modified Eagle's medium (DMEM; Welgene, Geyongsan, Korea) supplemented with glutamine $(2 \mathrm{mM})$, penicillin $(400 \mathrm{U} / \mathrm{ml})$, streptomycin $(50 \mathrm{mg} / \mathrm{ml})$, and $10 \%$ fetal bovine serum (FBS; Welgene) in a humidified $5 \% \mathrm{CO}_{2}$ atmosphere at $37^{\circ} \mathrm{C}$. Skin fibroblasts were used for the experiments at passages 6-8. For chemical treatment, skin fibroblasts were serum-starved for $24 \mathrm{~h}$ in DMEM containing 0.1\% FBS. This study was approved by the Institutional Review Board at Seoul National University Hospital and conducted according to the Declaration of Helsinki (IRB no. 1101-116-353).

Western blotting. The amounts of procollagen and MMP-1 proteins secreted into culture media were analyzed. $\beta$-actin was detected from equal volume of cell lysate as a loading control for procollagen and MMP-1. For the detection of p-ERK1/2, JNK, p38, I KB $\alpha$ and IRF3 in cell lysates, cells were washed twice with ice-cold phosphate buffered saline, and then lysed in radioimmunoprecipitation assay (RIPA) buffer (EMD Millipore). Cell lysates were incubated in RIPA buffer for $30 \mathrm{~min}$ at $4^{\circ} \mathrm{C}$, centrifuged for $15 \mathrm{~min}$ at $12,000 \mathrm{x} \mathrm{g}, 4^{\circ} \mathrm{C}$, and then supernatants were collected. Protein concentrations were measured by Bradford protein assay using the Bio-Rad protein assay (Bio-Rad Laboratories, Mississauga, ON, Canada). Protein $(30 \mu \mathrm{g})$ was separated by $10 \%$ SDS-PAGE. Separated proteins were transferred onto PVDF membranes, which were then incubated in blocking buffer consisting of 5\% skim milk in TBST at room temperaature for $30 \mathrm{~min}$. Then, membranes were incubated with the appropriate primary antibodies at $4^{\circ} \mathrm{C}$ for $16 \mathrm{~h}$ and secondary antibodies at room temperature for $1 \mathrm{~h}$. The signals were developed by enhanced chemiluminescence (GE Healthcare Life Sciences, Chalfont, UK). After being probed for procollagen and MMP-1, the same membrane was washed and stained with Coomassie Blue as a loading control. For certain experiments, the relative level of protein bands was quantified by densitometric analysis (ImageJ v1.47; National Institutes of Health, Bethesda, MD, USA).

Statistical analysis. Experiments were carried out in triplicate. Data are expressed as mean values \pm standard error of the mean. Statistical analysis was performed using the Student's t-test (Microsoft Office Excel 2013; Microsoft Corporation, Redmond, WA, USA). $\mathrm{P}<0.05$ was considered to indicate a statistically significant difference.

\section{Results}

Treatment of poly(I:C), but not Pam $3 C S K 4$, reduces procollagen expression in skin fibroblasts. Poly(I:C) is commonly known as a viral PAMP mimic (19). It has been demonstrated that poly(I:C) inhibits procollagen expression in skin fibroblasts (18). To confirm this result in the present study, various doses of poly(I:C) were administered to cultured skin fibroblasts. Following $48 \mathrm{~h}$ of treatment, the protein expression of procollagen was analyzed. It was identified that poly(I:C) reduced procollagen expression dose-dependently in skin fibroblasts (Fig. 1A). Next, in order to investigate whether other PAMPs or PAMP mimics can also inhibit procollagen expression levels in skin fibroblasts, skin fibroblasts were treated with a bacterial PAMP mimic, Pam3CSK4, which is commonly used as a ligand for TLR1/2 $(16,20)$. It was observed that treatment with $5 \mu \mathrm{g} / \mathrm{ml}$ Pam3CSK4 and $20 \mu \mathrm{g} / \mathrm{ml}$ poly(I:C) induced MMP-1 expression to a similar level. However, only poly(I:C), but not Pam3CSK4, reduced procollagen expression in skin fibroblasts (Fig. 1B). Thus, the data indicated that Pam3CSK4 has no effect on procollagen expression in skin fibroblasts.

Treatment of poly(I:C), but not Pam3CSK4, induces activation of IRF3 in skin fibroblasts. Since only poly(I:C), but not Pam3CSK4, reduced procollagen expression, it was hypothesized that specific signaling pathway(s) could be involved in poly(I:C)-induced procollagen reduction. Thus, 
A

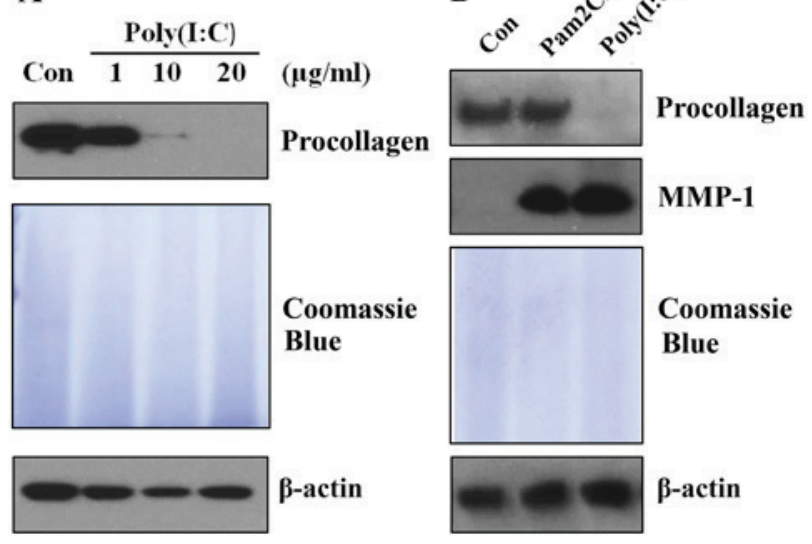

Figure 1. Treatment with poly(I:C), but not Pam3CSK4, reduces procollagen expression in skin fibroblasts. (A) Following serum-starvation, skin fibroblasts were incubated with various concentrations of poly(I:C) for $48 \mathrm{~h}$. (B) Skin fibroblasts were incubated with $5 \mu \mathrm{g} / \mathrm{ml} \mathrm{Pam3CSK} 4$ and $20 \mu \mathrm{g} / \mathrm{ml}$ poly(I:C) for $48 \mathrm{~h}$. Protein level of procollagen and MMP-1 in the cell culture medium and $\beta$-actin in the cell lysates were analyzed by western blotting, and total protein in the cell culture medium was analyzed by Coomassie Blue staining of the same membrane used to detect procollagen and MMP-1. The data presented are representative of three experiments. Con, control; MMP-1, matrix metalloproteinase-1

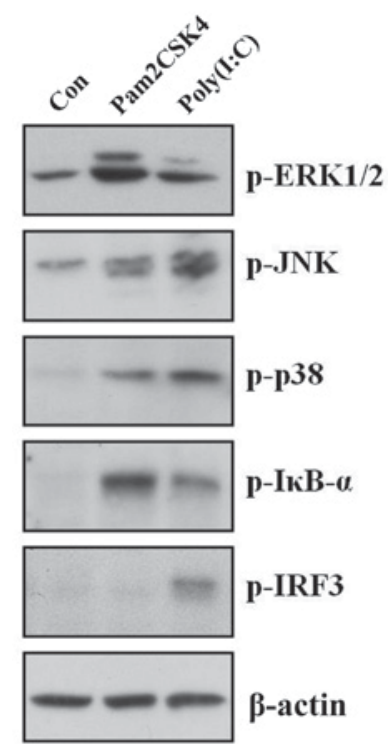

Figure 2. Treatment of poly(I:C), but not Pam3CSK4, induces activation of IRF3 in skin fibroblasts. Cultured skin fibroblasts were stimulated with $5 \mu \mathrm{g} / \mathrm{ml}$ Pam3CSK 4 and $20 \mu \mathrm{g} / \mathrm{ml}$ poly(I:C) for $2 \mathrm{~h}$. Cell lysates were fractionated by SDS-PAGE and then protein levels of MAPKs (p-ERK1/2, p-JNK, and p-p38), p-IкB- $\alpha$ and p-IRF3 were analyzed by western blotting. A single representative experiment is presented from three different experiments. Con, control; p, phosphorylated; ERK, extracellular signal-regulated kinase; JNK, c-Jun N-terminal kinase; IкB- $\alpha$, nuclear factor of $\kappa$ light polypeptide gene enhancer in B-cells inhibitor, $\alpha$; IRF3, interferon regulatory factor 3 .

the activation of several signaling pathways was checked and compared following treatment of poly(I:C) and Pam3CSK4. At $2 \mathrm{~h}$ following treatment, it was observed that Pam3CSK4 and poly(I:C) induced phosphorylation of ERK1/2, JNK, p38 and $\mathrm{I} \kappa \mathrm{B}-\alpha$. However, only poly(I:C), not Pam3CSK4, induced the phosphorylation of IRF3 (Fig. 2).
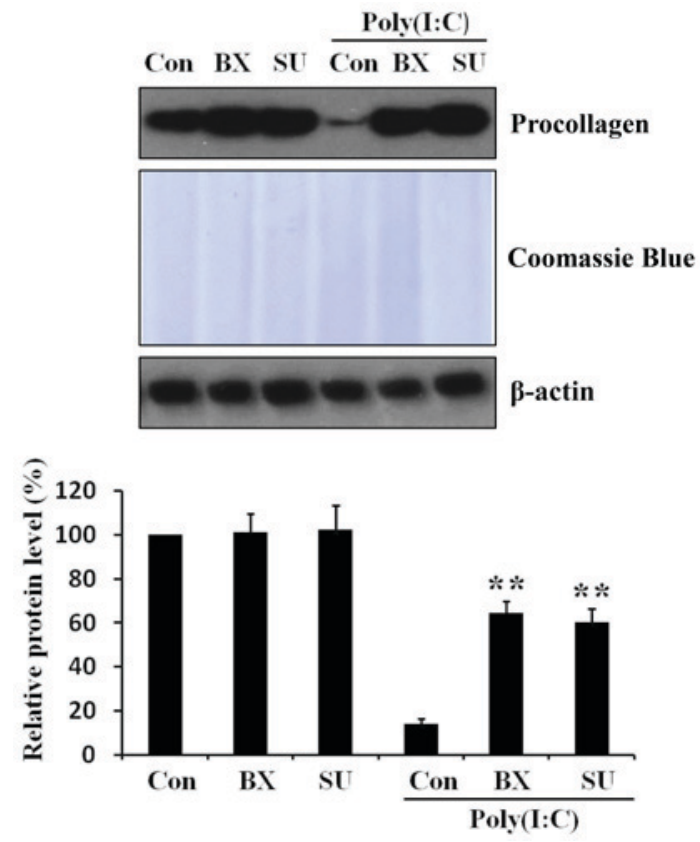

Figure 3. Inhibition of IRF3 signaling pathway mitigates poly(I:C)-induced procollagen reduction in skin fibroblasts. Following serum-starvation, skin fibroblasts were pretreated $1 \mathrm{~h}$ with two TBK1 inhibitors: $0.1 \mu \mathrm{M}$ BX795 and $10 \mu \mathrm{M}$ SU6668. The cells were then further incubated with $20 \mu \mathrm{g} / \mathrm{ml}$ poly(I:C) for $48 \mathrm{~h}$. The protein level of procollagen in the cell culture medium and $\beta$-actin in the cell lysates were analyzed by western blotting, and total protein in the cell culture medium was analyzed by Coomassie Blue staining of the same membrane used to detect procollagen. The data presented are representative of three independent experiments. Values are mean \pm standard error of the mean. ${ }^{* *} \mathrm{P}<0.01$ vs. poly(I:C)-treated control. IRF3, interferon regulatory factor 3; TBK1, Tank binding kinase 1; Con, control; BX, BX795; SU, SU6668.

Inhibition of IRF3 signaling pathway relieves poly(I:C)-induced procollagen reduction in skin fibroblasts. To investigate whether IRF3 signaling pathway is involved in the reduction of procollagen by $\operatorname{poly}(\mathrm{I}: \mathrm{C})$ in skin fibroblasts, cells were pretreated for $1 \mathrm{~h}$ with two different TBK1 (an upstream molecule of IRF3) inhibitors: BX795 (21) and SU6668 (8). Then poly(I:C) was further added to the cell cultured medium. After $48 \mathrm{~h}$, it was observed that pretreatment of IRF3 pathway inhibitors mitigated poly(I:C)-induced procollagen reduction (Fig. 3). Thus, the results indicated that poly(I:C)-induced procollagen reduction is regulated by IRF3 signaling pathways in skin fibroblasts.

\section{Discussion}

dsRNA can act as a form of genetic information carried by certain viruses including retroviruses (22). dsRNA can also exist as an endogenous TLR3 ligand. For example, it was identified that UV-damaged self-noncoding RNA can be detected by TLR3 (23). Poly(I:C) is a synthetic dsRNA which has frequently been used as a representative dsRNA ligand in a number of studies $(14,15)$. The effects of poly(I:C) on collagen expression have been extensively investigated in a number of studies $(15,18)$ and it has been demonstrated that subcutaneous poly(I:C) delivery by osmotic pumps induces epidermal hyperplasia and increased matrix deposition in mice. TGF- $\beta$-related genes were elevated in lesional mouse 
skin and Farina et al (14) concluded that chronic TLR3 stimulation can induce cutaneous fibrosis in mice. However, other studies have provided evidence of the anti-fibrosis effects of poly(I:C) in mice. For example, studies have identified that injection of poly(I:C) ameliorated lung and liver fibrosis in mice $(24,25)$. Conflicting results of poly(I:C) on collagen expression were also identified in several in vitro studies $(15,18)$. Sugiura et al $(15)$ identified that activation of TLR3 by poly(I:C) augments collagen production in cultured human fetal lung fibroblasts. A NF-кB-TGF- $\beta 1$-dependent pathway was identified to be involved in the processes. It has been suggested (15) that IRF3 signaling pathway is not associated with collagen production by poly(I:C) in fetal lung fibroblasts. Another study (18) demonstrated that poly(I:C) reduces TGF- $\beta$-induced collagen expression in cultured skin fibroblasts. It was suggested that poly(I:C) upregulates the expression of Smad7 which inhibits TGF- $\beta$-induced collagen production.

The present study identified that treatment with poly(I:C), but not another PAMP, Pam3CSK4, inhibited procollagen expression in skin fibroblasts (Fig. 1). It was hypothesized that poly(I:C) could activate specific factor(s) that mediate(s) procollagen reduction in skin fibroblasts. Indeed, poly(I:C), but not Pam3CSK4, induced activation of IRF3 (Fig. 2). Apart from the IRF3 pathway, the other signaling pathways, including MAPKs and NF- $\mathrm{KB}$ pathways, were activated by treatment with the two PAMPs (Fig. 2). To understand the role of IRF3 in poly(I:C)-induced collagen reduction, two inhibitors were used for the IRF3 pathway (Fig. 3). The data indicated that poly(I:C)-induced procollagen reduction is regulated by IRF3 pathway in skin fibroblasts (Fig. 3). At present, only a small number of studies have investigated the relationship between IRF3 and collagen. It has been suggested that the IRF3 pathway is not associated with increased collagen production by poly(I:C) in cultured fetal lung fibroblasts (15). Recently (26), it was demonstrated that inhibition of IRF3 significantly decreases the expression of type I collagen in human hepatic stellate cells: Indeed, overexpression of IRF3 increases collagen expression. The data appears to conflict with the data of the present study, which demonstrated that activation of IRF3 decreased collagen expression. However, another study by Xu et al (27) demonstrated that poly(I:C) suppresses TGF- $\beta$-induced Smad3 signaling through activation of IRF3 in human HepG2 hepatoma cells. This result may imply that poly(I:C) can suppress TGF- $\beta$-induced collagen expression through IRF3 in human HepG2 hepatoma cells, although the direct evidence was not revealed (27). Thus, the data from the present study and the published results may indicate that IRF3 pathway can be either a suppressor or inducer of collagen production depending on experiment conditions.

In conclusion, it has been demonstrated that the IRF3 signaling pathway is involved in poly(I:C)-induced procollagen reduction in skin fibroblasts. In particular, it is for the first time, to the best of the authors' knowledge, demonstrated that activation of the IRF3 signaling pathway is involved in procollagen reduction in skin fibroblasts. Understanding the relation between IRF3 and collagen may aid the treatment of fibrotic diseases, including scleroderma and liver fibrosis.

\section{Acknowledgements}

The present study was supported by a grant from the Korea Health Technology R\&D Project through the Korea Health Industry Development Institute, funded by the Ministry of Health and Welfare, Republic of Korea (grant no. HI14C1277) and by a grant from the National Research Foundation of Korea funded by the Ministry of Science, ICT and Future Planning (grant no. 2014M3C9A2064536).

\section{References}

1. Chen K, Huang J, Gong W, Iribarren P, Dunlop NM and Wang JM: Toll-like receptors in inflammation, infection and cancer. Int Immunopharmacol 7: 1271-1285, 2007.

2. Ermertcan AT Öztürk F and Gündüz K: Toll-like receptors and skin. J Eur Acad Dermatol Venereol 25: 997-1006, 2011.

3. Medzhitov R: Toll-like receptors and innate immunity. Nat Rev Immunol 1: 135-145, 2001.

4. Miller LS and Modlin RL: Toll-like receptors in the skin. Semin Immunopathol 29: 15-26, 2007.

5. Takeuchi O, Kaufmann A, Grote K, Kawai T, Hoshino K, Morr M, Mühlradt PF and Akira S: Cutting edge: Preferentially the R-stereoisomer of the mycoplasmal lipopeptide macrophage-activating lipopeptide- 2 activates immune cells through a toll-like receptor 2- and MyD88-dependent signaling pathway. J Immunol 164: 554-557, 2000.

6. Takeuchi O, Sato S, Horiuchi T, Hoshino K, Takeda K, Dong Z, Modlin RL and Akira S: Cutting edge: Role of Toll-like receptor 1 in mediating immune response to microbial lipoproteins. J Immunol 169: 10-14, 2002.

7. Takeuchi O, Kawai T, Mühlradt PF, Morr M, Radolf JD, Zychlinsky A, Takeda K and Akira S: Discrimination of bacterial lipoproteins by Toll-like receptor 6. Int Immunol 13: 933-940, 2001.

8. Kalali BN, Köllisch G, Mages J, Müller T, Bauer S, Wagner H, Ring J, Lang R, Mempel M and Ollert M: Double-stranded RNA induces an antiviral defense status in epidermal keratinocytes through TLR3-, PKR-, and MDA5/RIG-I-mediated differential signaling. J Immunol 181: 2694-2704, 2008.

9. Tanner NK and Linder P: DExD/H box RNA helicases: From generic motors to specific dissociation functions. Mol Cell 8: 251-262, 2001.

10. Sloane JA, Blitz D, Margolin Z and Vartanian T: A clear and present danger: Endogenous ligands of Toll-like receptors. Neuromolecular Med 12: 149-163, 2010.

11. Lester SN and Li K: Toll-like receptors in antiviral innate immunity. J Mol Biol 426: 1246-1264, 2014.

12. Pattanaik D, Brown M, Postlethwaite BC and Postlethwaite AE: Pathogenesis of systemic sclerosis. Front Immunol 6: 272, 2015.

13. Rittié L and Fisher GJ: UV-light-induced signal cascades and skin aging. Ageing Res Rev 1: 705-720, 2002.

14. Farina GA, York MR, Di Marzio M, Collins CA, Meller S, Homey B, Rifkin IR, Marshak-Rothstein A, Radstake TR and Lafyatis R: Poly(I:C) drives type I IFN- and TGF $\beta$-mediated inflammation and dermal fibrosis simulating altered gene expression in systemic sclerosis. J Invest Dermatol 130: 2583-2593, 2010.

15. Sugiura H, Ichikawa T, Koarai A, Yanagisawa S, Minakata $Y$, Matsunaga K, Hirano T, Akamatsu K and Ichinose M: Activation of Toll-like receptor 3 augments myofibroblast differentiation. Am J Respir Cell Mol Biol 40: 654-662, 2009.

16. Lee Y, Kim H, Kim S, Kim KH and Chung JH: Activation of toll-like receptors 2, 3 or 5 induces matrix metalloproteinase-1 and -9 expression with the involvement of MAPKs and NF-kappaB in human epidermal keratinocytes. Exp Dermatol 19: e44-e49, 2010.

17. Reimer T, Schweizer M and Jungi TW: Stimulation-specific contribution of p38 and JNK to IFN-beta gene expression in human macrophages. J Interferon Cytokine Res 27: 751-755, 2007.

18. Fang F, Ooka K, Sun X, Shah R, Bhattacharyya S, Wei J and Varga J: A synthetic TLR3 ligand mitigates profibrotic fibroblast responses by inducing autocrine IFN signaling. J Immunol 191: 2956-2966, 2013. 
19. Kinnier CV, Martinu T, Gowdy KM, Nugent JL, Kelly FL and Palmer SM: Innate immune activation by the viral PAMP poly $\mathrm{I}: \mathrm{C}$ potentiates pulmonary graft-versus-host disease after allogeneic hematopoietic cell transplant. Transpl Immunol 24: 83-93, 2011.

20. Yao C, Oh JH, Lee DH, Bae JS, Jin CL, Park CH and Chung JH: Toll-like receptor family members in skin fibroblasts are functional and have a higher expression compared to skin keratinocytes. Int J Mol Med 35: 1443-1450, 2015.

21. Yao C, Lee DH, Oh JH, Kim MK, Kim KH, Park CH and Chung JH: Poly(I:C) induces expressions of MMP-1, -2, and -3 through various signaling pathways including IRF3 in human skin fibroblasts. J Dermatol Sci 80: 54-60, 2015.

22. Chiappinelli KB, Strissel PL, Desrichard A, Li H, Henke C Akman B, Hein A, Rote NS, Cope LM, Snyder A, et al: Inhibiting DNA methylation causes an interferon response in cancer via dsRNA including endogenous retroviruses. Cell 169: $361,2017$.
23. Bernard JJ, Cowing-Zitron C, Nakatsuji T, Muehleisen B, Muto J, Borkowski AW, Martinez L, Greidinger EL, Yu BD and Gallo RL: Ultraviolet radiation damages self noncoding RNA and is detected by TLR3. Nat Med 18: 1286-1290, 2012.

24. Hyde DM and Giri SN: Polyinosinic-polycytidylic acid, an interferon inducer, ameliorates bleomycin-induced lung fibrosis in mice. Exp Lung Res 16: 533-546, 1990.

25. Hou X, Yu F, Man S, Huang D, Zhang Y, Liu M, Ren C and Shen J: Polyinosinic-polycytidylic acid attenuates hepatic fibrosis in C57BL/6 mice with Schistosoma japonicum infection. Acta Trop 121: 99-104, 2012.

26. Ni MM, Xu T, Wang YR, He YH, Zhou Q, Huang C, Meng XM and Li J: Inhibition of IRF3 expression reduces TGF- $\beta 1$-induced proliferation of hepatic stellate cells. J Physiol Biochem 72: 9-23-2016.

27. Xu P, Bailey-Bucktrout S, Xi Y, Xu D, Du D, Zhang Q, Xiang W, Liu J, Melton A, Sheppard D, et al: Innate antiviral host defense attenuates TGF- $\beta$ function through IRF3-mediated suppression of Smad signaling. Mol Cell 56: 723-737, 2014 